\title{
Efficient Approach for Designing Gesture Controlled Robotic Arm
}

\author{
Shivani, Shagun Gaur,Paresh Khaneja,Rashmi Sharma,Simranpreet \\ Kaur,Mehakpreet Kaur \\ Department of ECE \\ Chitkara University, \\ Chandigarh, India \\ shalu16sharma@gmail.com, shagungaur74@yahoo.co.in, \\ Pareshkhaneja@gmail.com,rashmisharma1505@gmail.com, \\ simranchhabra2394@gmail.com,mehakpreet_26july@yahoo.com
}

\begin{abstract}
Gesture Controlled Robot is a robot that can be moved according to our limb movements. All we need to do is just wear a gesture device which consists of a sensor such as accelerometer. The sensor mounted on your hand will judge the movement of hand in a particular direction which will result in the movement of the robot in the direction of the hand movement. In this era of modern technology, technology has decreased working hours and has made complicated operations more effortless. Robotics is a very vast field that has helped us make some wonderful machines that assist us in our daily lives or even commercially. Typical industrial robots usually perform tasks that are difficult, dangerous, too complex or dull. They lift heavy weights, and a lot of manual work such as welding can be done using robots. They can perform the same course of action in lesser time with more accuracy. This robotic arm is focused on developing a device by interfacing the hardware with software and creating something which makes the performance of complicated activities comfortable. Though the working of a robotic arm is very simplified, the implementation of this arm requires a good knowledge of Engineering Mechanics, Arduino platform, Electronics Devices mainly sensors and Embedded Systems as well.
\end{abstract}

Keywords: Gesture, Robotic Arm, Accelerometer, Servo Motor, Microcontroller, Sensors

\section{Introduction}

When humans started wishing to become God, they made robots in order to compete with God. When we try to design robot as an alternative for a human being, we have to visualize the brain of a human with software and also simulate the organs of humans with mechanical equipments. Gestures are and have been the most integral part of human conversations. They convey a lot more than words can and are not just limited to human interactions but are also used to interact with the digital world around us as well. So we decided to develop something that breaks the barriers and brings the digital world closer to the human world using gestures. The name of our integrated project is gesture controlled robotic arm. The main objective behind this project is to make a robotic arm that would imitate the gestures of a human arm. There are three parts that constitute our arm. The arm moves in accordance with the motion of the glove that is worn on the human arm. In this world there are differently disabled people who face difficulty in their daily life. There are many handicapped people who are not able to perform their daily tasks. To make their life easy and comfortable, robots play a very powerful role. With the 
help of gestures handicapped people can do their work by just giving instructions to the robotic arm as the gripper attached to the arm can lift the heavy weights also depending on capability of gripper. Robot is a machine which can perform human tasks with maximum accuracy with zero human error. Not only can they help amputees but the areas which are hazardous or risky for human life such as harmful gas industries and places where explosive gases exist, gesture based arm makes it easier and reduces the risk. A gesture controlled robot can be controlled with the movement of hand only. It works as per instructions given by the operator through gestures. Hand glove consists of sensors and microcontroller.

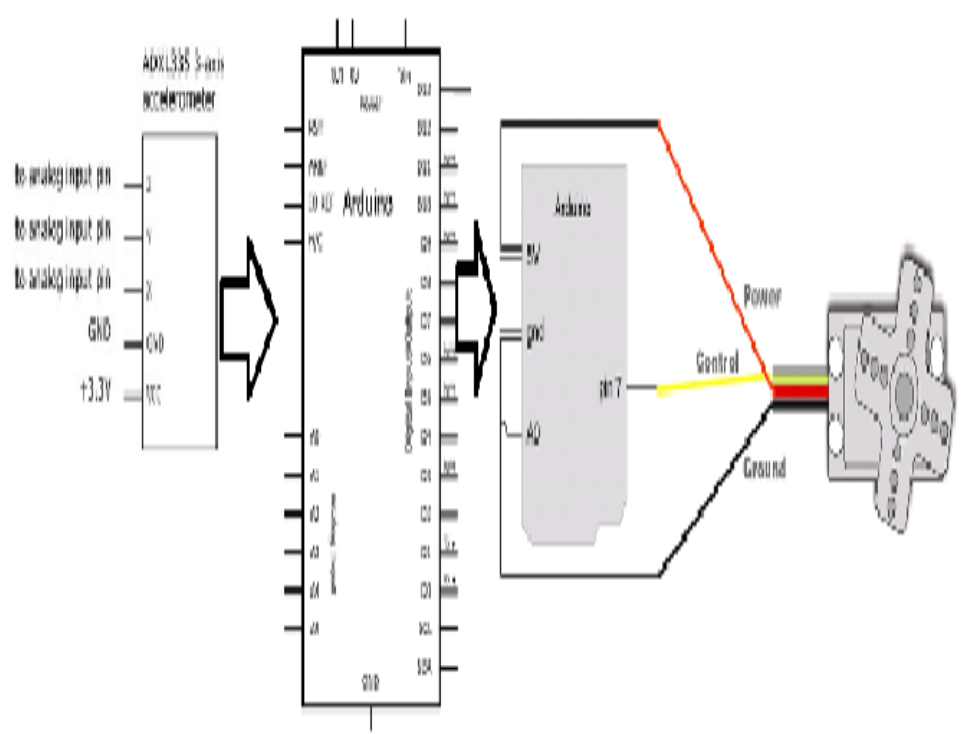

Figure 10. Circuit Diagram of Robotic Arm

\section{Related Work}

Today there are a lot of robotic arms that are controlled using human gestures. In this column, we highlight some of the already existing robotic arms and their basic features. Robotics is a very vast field with its focus concentrated on controlling things automatically. In this section, we also try to differentiate our project from the other robotic arms that already have been talked about.

\subsection{Design and Implementation of a Wireless Gesture Controlled Robotic Arm with Vision [9]}

In this particular project, the robotic arm has gripping capability. They have made use of a camera which sees the arm movement and recognizes the predefined movements and moves the robotic arm accordingly [8]. This is, however, a more complex version of controlling an arm with gestures. In our project, we have made a glove which has the sensor fixed on it so there is no camera involved, thus, less complexity and less chances of error. Also, the above said project has used an accelerometer as their sensor as opposed to ours. We have made use of a gyroscope which provides even the horizontal motion which is restricted in an accelerometer.

\subsection{Gyro-Accelerometer Based Control of a Robotic Arm Using AVR Microcontroller [10]}

The above said robotic arm is a gyro-accelerometer based project in which they have used AVR microcontroller and the arm can lift objects. In our project, 
however, we have made an addition to the normal robotic arm with a gripper by adding a full-fledged hand to it which contains four fingers and a thumb. In addition to picking things up, it can also show different combination of fingers or different gestures similar to a human arm.

\subsection{Accelerometer based Control of an Industrial Robotic Arm [7]}

In this project, they have used a neural network that uses a back propagation algorithm to recognize arm gestures which are later used as the input to control the robot. The sensor used by them is accelerometer. But accelerometer often poses a big problem when the arm is made to move about a single axis. In that scenario, the accelerometer readings don't change. Therefore, we used gyroscope because the coriolis force enables the rotator motion even if an object is moving around its own axis.

\subsection{GripSee: A Gesture-Controlled Robot for Object Perception and Manipulation [6]}

In this project, they have enabled the interaction of the computer with the robotic arm. The circuit uses accelerometer for the gestures but according to our study, an accelerometer can detect only two type of motions, pitch and roll motions respectively. When it comes to the yaw motion, the value of acceleration due to gravity did not change in this motion. So for yaw motion, we have used accelerometer plus gyroscope module that is MPU6050. MPU6050 can give readings for yaw, pitch as well as roll motion.

\section{Working:}

The working of our gesture controlled arm is

\subsection{Detecting Movements}

Ideally, to make such a gesture controller that is controlling an arm using gestures, flex sensors are used to detect the movement but because they are costly and delicate too, we decided to use sliding linear potentiometers for this purpose. We attached a string to the knob of the sliding potentiometer, and tied the string to the fingers so now when we bend our fingers forward, the knob of the potentiometer pushed forward suddenly. We attached a string to the pot so that the knob goes back when we released our fingers.

\subsection{Caliberating the Accelerometer}

We used a standard ADXL335 three axis accelerometer which detects position and movement and tried to calibrate it. When we tested it for a single axis, it worked accurately. The code is quite easy. We took the minimum and maximum readings interpreted from the accelerometer and mapped it from 0 to 180. We then assigned these values to the servo motor. But when we tried using two axis of accelerometer simultaneously, a lot of errors started coming up.

The $\mathrm{z}$-axis reading did not change at all, no matter how we moved the accelerometer. The $\mathrm{x}$-axis reading changed when the accelerometer was rotated about $\mathrm{x}$-axis but it also changed when we rotated it about the y-axis. The y-axis worked correctly. So we decided to use y-axis for the purpose of detection of elbow movement. The Accelerometer has 6 pin as shown in Figure 2. 


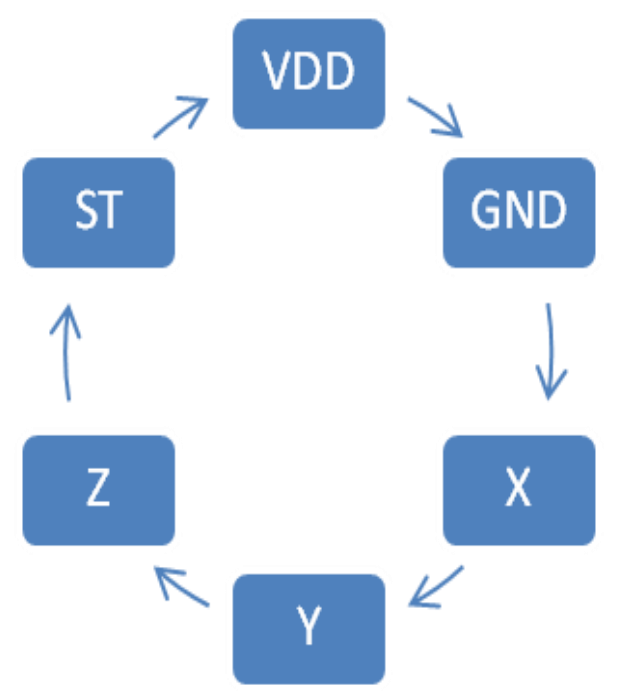

Figure 2. Pin of Accelerometer

We will provide the +5 volt to VDD and simply connect GND to the ground for biasing. On $\mathrm{X}$, we will receive the analog data input for $\mathrm{x}$ direction movement. On $\mathrm{Y}$, we will receive the analog data input for $\mathrm{y}$ direction movement. And on $\mathrm{Z}$, we will receive the analog data for $\mathrm{z}$ direction movement. ST pin is required for setting the sensitivity of the accelerometer.

\subsection{Mechanical Work}

The material used for making the arm is wood. This is because wood is quite light in weight and moves with precision as well. We used servos for the motion of the arm and also attached a servo to the gripper along with a dc motor. The base was made to rotate by affixing it with a wooden block in which a servo was embedded.

\subsection{Robotic Arm}

The advantage of using a motion sensor such as gyroscope leads to an easy control of our robotic arm. The use of complicated mechanisms like gears or press buttons can be avoided. Applications are performed with full accuracy with very less probability of error. This level of precision is very difficult to attain with any other system. Robots are efficient in increasing throughput speed that directly have an impact on production as they can work at a uniform speed without stopping for breaks and have great capability to produce more efficiency than human beings. Robots also bring about an increase in workplace safety.

This is the vital part of the system since this part which does the picking and dropping task of the project. The robotic arm is equipped with a gripper for picking and placing the objects and an arm mounted on a robot car for raising and lowering the objects. Both the arm and gripper are attached with servo motor to control the movement. These movements are synchronized with the hand gestures of the user, therefore, operating the robotic Arm. The accelerometer mounted on hand which interprets the hand gestures. Also, the different hand gestures are described below:

GESTURE 1: To lower the arm

GESTURE 2: To raise the Arm

GESTURE 3: To close the Gripper mouth so that it can pick the object

GESTURE 4: To open the Gripper Mouth so that it can place / drop the object 


\section{Components Used}

\subsection{List of Components}

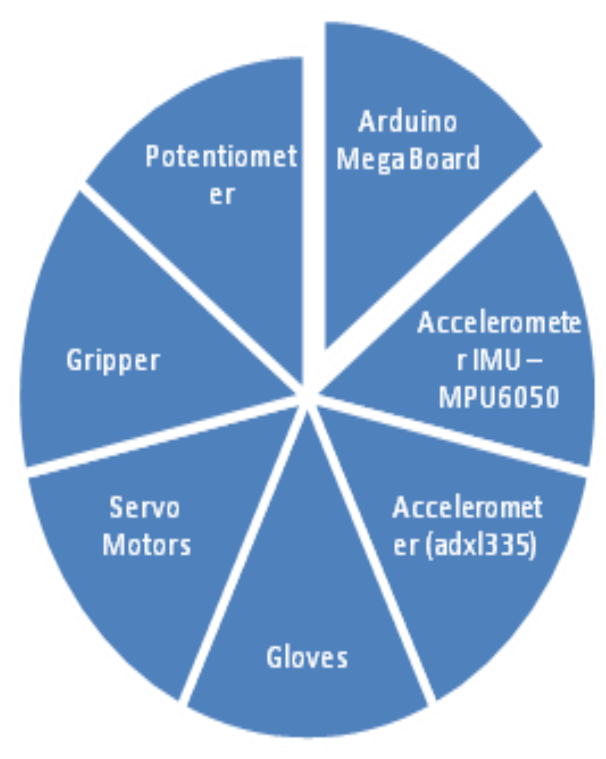

Figure 3. Components Used

\subsection{Component Description}

\subsubsection{Arduino UNO Board}

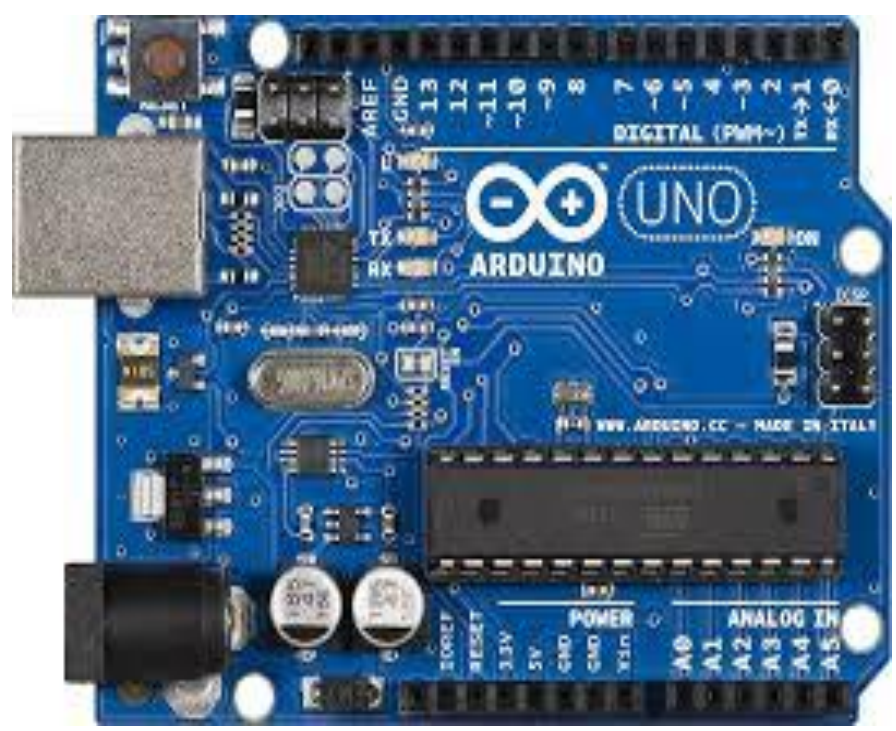

Figure 4. Schematic of Arduino UNO Board [1]

It contains Atmega AVR328 micro controller and has fourteen digital Output and Input pins out of which 6 pins can be used as PWM outputs and 6 pins can be used as analog inputs, ceramic resonator, a USB connection, a power jack, an ICPS header, and a reset button. The operating voltage is of UNO board is $5 \mathrm{~V}$. 
Table 1. Specifications of Arduino Board

\begin{tabular}{|l|l|}
\hline Operating Voltage & $5 \mathrm{~V}$ \\
\hline Input Voltage (recommended) & $7-12 \mathrm{~V}$ \\
\hline Input Voltage (limits) & $6-20 \mathrm{~V}$ \\
\hline Digital I/O Pins & 14 (of which 6 provide PWM output) \\
\hline Analog Input Pins & 6 \\
\hline DC Current per I/O Pin & $40 \mathrm{Ma}$ \\
\hline DC Current for 3.3V Pin & $50 \mathrm{~mA}$ \\
\hline Flash Memory & $32 \mathrm{~KB}$ of which $0.5 \mathrm{~KB}$ \\
\hline SRAM & $2 \mathrm{~KB}$ \\
\hline EEPROM & $1 \mathrm{~KB}$ \\
\hline Clock Speed & $16 \mathrm{MHz}$ \\
\hline Length & $68.6 \mathrm{~mm}$ \\
\hline Weight & $25 \mathrm{~g}$ \\
\hline
\end{tabular}

\subsubsection{Gyro, Accelerometer Imu - Mpu6050}

The 6 degree of freedom gyro, accelerometer is designed for low cost and consumes less power. They can measure gravitational forces, craft velocity and also the orientations. In our project, we have used gyroscope module because accelerometer can't give the readings of three motions. One, when the accelerometer is parallel to earth, the value of acceleration due to gravity will not change. That's why instead of using accelerometer alone, we have used gyroscope, which is a combination of accelerometer and gyroscope and magnetometer.

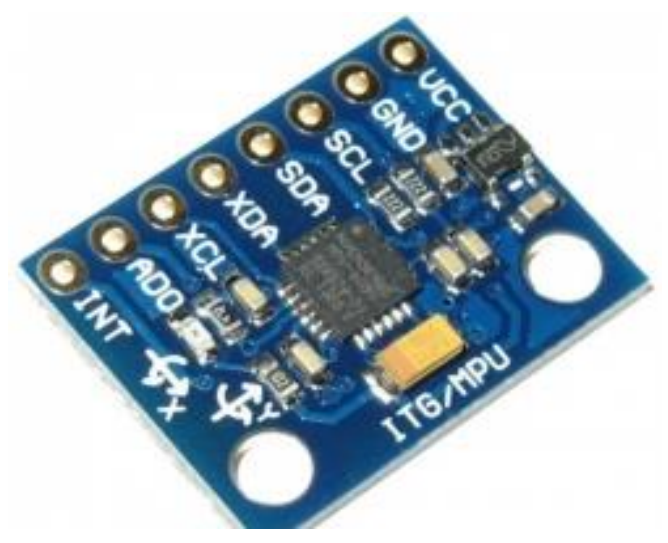

Figure 5. Schematics of MPU6050 Board [4]

Reading the default values for the gyroscope is very easy. The DMP i.e. Digital Motion Processor can do quick calculations on the chip. This reduces the load for the microcontroller and arduino. The DMP is also able to perform calculations with the sensor values of another chip, for example a magnetometer connected to the second -I2C-bus. The measurements should be done in these conditions:

- The sensor should be placed as horizontal as possible.

- It should ideally be placed on concrete instead of a wooden table.

3-axis accelerometer is a sensor that can measure the movement affecting the sensor in all the 3 directions $\mathrm{x}, \mathrm{y}, \mathrm{z}$ axis. Sensitivity of accelerometer is very high and are very difficult to manage.ADX1335 is ratio metric device, that is, its output sensitivity depends on input voltage. For managing the problem of sensitivity we 
can use the "for" loop in the program and take average values. The main advantage of the accelerometer is that the values will not change unless there is a change in position or it detects any sort of movement. But the disadvantage of an accelerometer is that any sort of discrepancy or disturbance can trigger an error in the readings. Therefore, to make these values correct and accurate, gyroscope sensor was used.

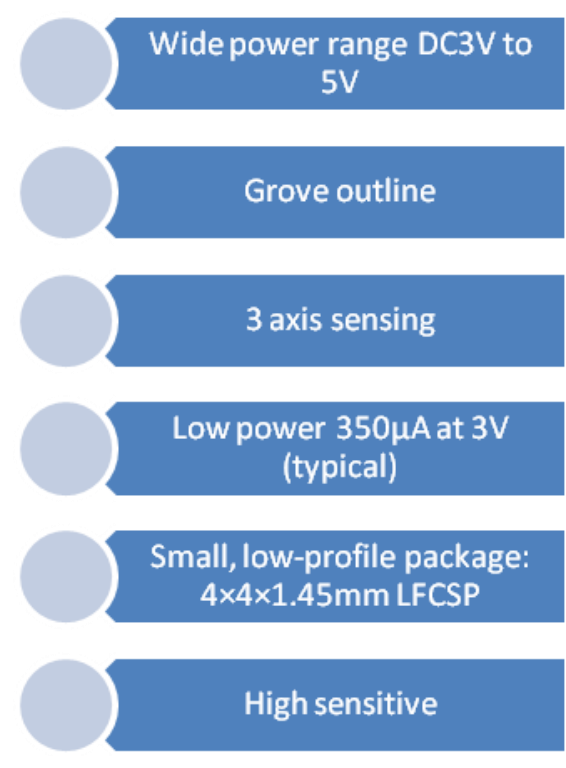

Figure 6. Features of ADXL335

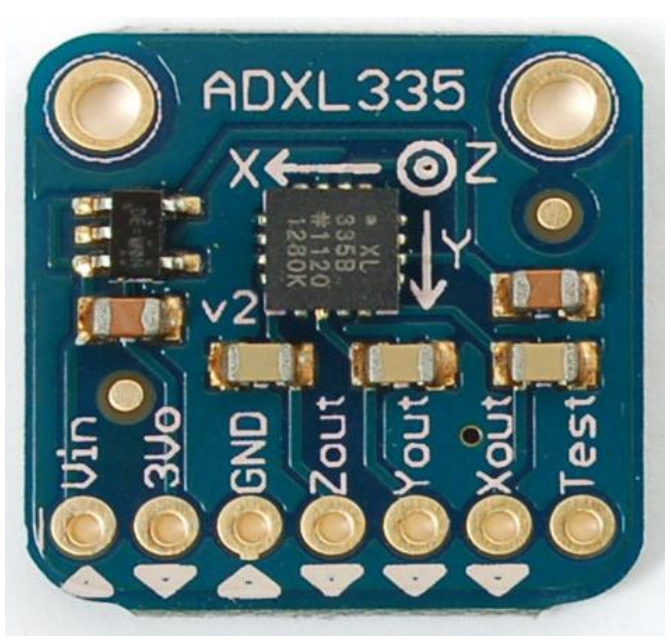

Figure 7. Schematic of ADXL335 [5]

\subsubsection{Gloves}

Robotic glove houses the circuit which controls the robotic arm. It consists of Arduino Mega 2560 .The Gyroscope installed takes the angles and acceleration in all three directions of the hand respectively, sends the signals to the Arduino Mega via wires where the values are combined and processed simultaneously. At the same time the potentiometers are doing their job by sending the degree of movement of the finger to the arduino mega. The processed values are then transmitted from the module to the robotic arm. The module takes the feedback from the arm and sends the new processed signals to it. 


\subsubsection{Servo Motors}

Servo motors have an error detecting feedback controlling system which controls the performance of a system. Servo motors are basically DC motors with a servo mechanism for precise and accurate control of angular positions. The servo motor's rotation is restricted from limit $90^{\circ}$ to $180^{\circ}$ but servos do not rotate continually and they can be used for rotations of 0 to $90^{\circ}$ or for 0 to $180^{\circ}$.

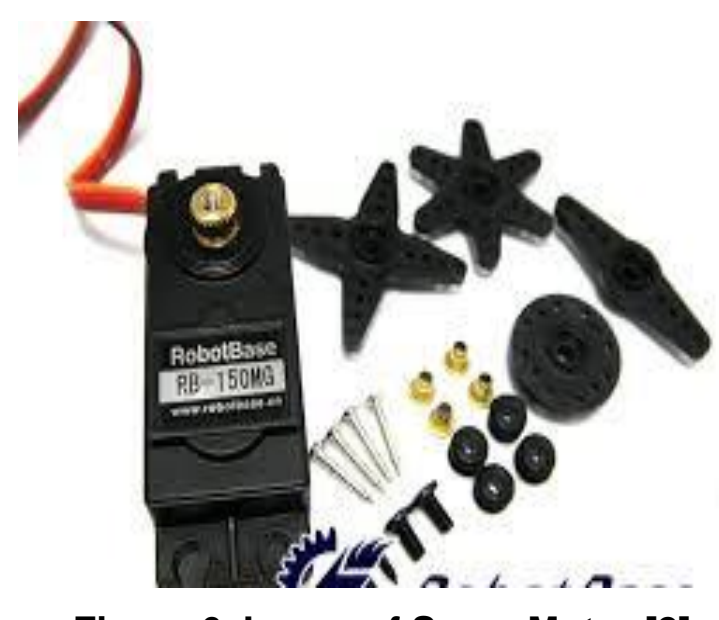

Figure 6. Image of Servo Motor [2]

We used three servo motors in our project. The first servo was embedded in the wooden base than acts as shoulder. The second servo motor was attached to the connecting joint between the shoulder and the elbow which helps in the horizontal movement of the whole system. The third servo motor was attached to the gripper that controls the opening and closing of its jaws.

\subsubsection{Gripper}

A mechanical gripper is a device that uses mechanical fingers controlled by a mechanism to hold an object. The fingers also referred to as the jaws of the gripper are addition to the main construct that actually make contact with the object either by physically or by grasping the object. The movement of sliding pot to a particular resistance in one direction would force the gripper to open, while the movement of the pot in opposite direction causes the spring to force the gripper to close.

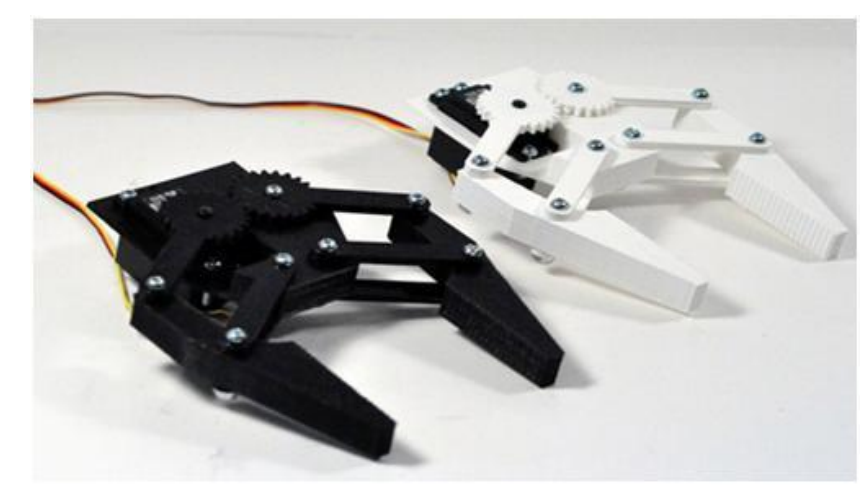

Figure 7. Image of Gripper [3] 


\subsubsection{Sliding Potentiometer}

Normally for making a controller that is capable of detecting finger movements, flex sensors are used but as they are extremely costly and breakable so we came up with the idea of using sliding pots to do that. These potentiometers are 8 times cheaper and far more accurate then the flex sensors.

\section{Conclusion}

The objective of this project is to make a robotic arm that can help disabled people or can be used at the places which are dangerous for human beings. The movement is precise, accurate, as well easy to control and friendly to use. The robotic arm has been made very carefully and in a detailed manner so that the movement of the robot can be controlled accurately. This robotic arm control method will be helpful in many aspects to make human life comfortable and easy.

\section{Future Scope}

The project is built on a wired model. It could further be developed to work on wireless communication, thus allowing the user to move in an even and an easier and an unrestricted manner.

1. Degrees of freedom of the robotic arm can be increased by implanting more servos motors.

2. Inverse kinematics technique can also be implemented in robotic arm.

3. Graphical user interface can also be added to make robotic arm user friendly.

4. With the help of web interface the arm can also be controlled in remote areas.

\section{References}

[1] Arduino Uno board Available at: http://brianeisenhauer.com/electronics/arduino-uno-in-hand-equalsproject-time/.

[2] Servo motor Available at: http://robotbase.en.alibaba.com/product/381216656211878901/RB_150MG_Servo_Motor_of_180_Degree_for_Robotic_Arm.html.

[3] Gripper Available at: http://www.kwartzlab.ca/2012/05/3d-printed-robot-gripper/

[4] Gyroscope Available at: http://playground.arduino.cc/Main/MPU-6050.

[5] Accelerometer Available at: http://www.instructables.com/id/Interfacing-ADXL335-with-ARDUINO/.

[6] M. Becker, "GripSee: A gesture-controlled robot for object perception and manipulation", Autonomous Robots, vol. 6, no. 2, (1999), pp. 203-221.

[7] J. N. Pires Neto and A. P. Moreira, "Accelerometer-based control of an industrial robotic arm", The 18th IEEE International Symposium on. Robot and Human Interactive Communication, RO-MAN 2009, IEEE, (2009).

[8] D. James and M. Shah, "Recognizing hand gestures", Springer Berlin Heidelberg Computer VisionECCV'94, (1994), pp. 331-340.

[9] L. Aggarwal, V. Gaur and P. Verma, "Design and Implementation of a Wireless Gesture Controlled Robotic Arm with Vision", International Journal of Computer Applications (0975 - 8887), vol. 79, no 13, (2013) October.

[10] B. A. Islma and T. C. Mallick, "Gyro-accelerometer based control of a robotic arm using AVR microcontroller”, 9th IEEE International forum on Strategic Technology (IFOST), (2014). 
International Journal of Control and Automation Vol. 8, No. 6 (2015) 